\title{
Mathematical analysis of experimental results in polycrystalline shape memory samples subject to a simple uniaxial tension test
}

\author{
$\underline{\text { J. Cortés-Pérez }}{ }^{\mathrm{a}, 1}$, J. G. González R. ${ }^{2}$, J. Carrera B. ${ }^{3}$ and H. Flores $Z^{4}$ \\ ${ }^{1}$ Centro Tecnológico Aragón, FES Argón, UNAM. Av. Rancho Seco s/n, Col. Impulsora, Cd. \\ Nezahualcoyotl, Edo. de México, México, C. P. 57130. \\ ${ }^{2}$ Instituto de Investigaciones en Materiales, UNAM. México D. F. México. \\ ${ }^{3}$ Departamento de Mecánica, DEPFI, UNAM. México D. F. México \\ ${ }^{4}$ Centro de Investigaciones en Materiales Avanzados. SEP-Conacyt. Chihuahua, Chihuahua. México.
}

\begin{abstract}
A set of experimental results reported in the literature was analysed by the application of a mathematical model developed previously. The experimental results analysed consist in a set of shape memory polycrystalline samples which was subject to simple uniaxial tension test. The crystalline orientation of several grains of the polycrystalline samples was measured and the variant formed was determined by the maximum Schmid factor criteria. Also the stress tensor was measure by the application of X-ray diffraction in situ technique. The information of the experimental study was employed for simulated the material behavior by the application of a mathematical procedure previously developed. A full analysis of the same experimental results was done and compared with them. The analysis procedure consists in generate for the 24 martensite variants: a) the distortion on the sample surface, b) the displacement field and c) The distortion of a mark on the sample's surface. Also the plane stress stated transformational diagram was employed to stress state analysis. The theoretical analysis shown a total characterization of the material behavior and therefore several important features during the stress induced martensite transformation as the identification of the variants formed in each grain with good agreement and the possibility of the apparition of other tensor stress components. Also, the simulation would be useful for the strain compatibility problem.
\end{abstract}

\section{Introduction}

It is clear that the study of the polycrystalline Shape Memory Materials SMM is very important for increase the performance of applications of these materials. As is well known, the high anisotropy of these materials and possible crystalline texture results in very complex to predict their global behaviour. A special attention requests the strain compatibility phenomena in a very high anisotropic polycrystalline material which suffer so large strain during the martensite transformation like the SMM.

About this problem, recently a experimental study about the mechanical behavior of polycrystalline Shape Memory Alloys (SMA) subject to a simple tension tests was reported [1]. The crystalline orientation of several grains of the polycrystalline samples was measured and the variant formed was determined by the maximum Schmid factor criteria. Also the stress tensor was measure by the application of X-ray diffraction in situ technique. Although these results are a very important contribution over the known of the grain to grain behaviour and their relation whit the global behaviour it is convenient subject them to a other analysis which allows us determinate others effect like the possible presence of stress related to near neighbor. Also the study of these results by image's analysis could represent a less expensive alternative to this analysis.

\section{Development}

First employing the transformation system elements were calculated the parameters reported for Kaouache et al[1] as: the Schmid Factor and the intersection of the habit plane and the observation surface. The comparison of these results with these reported by Kaouache et al [1] and we found next inconsistent:

aemail: jacop@correo.unam.mx

This is an Open Access article distributed under the terms of the Creative Commons Attribution-Noncommercial License (http://creativecommons.org/licenses/by-nc/3.0/), which permits unrestricted use, distribution, and reproduction in any noncommercial medium, provided the original work is properly cited. 
a) In general, the "Schmid Factor" values reported by Kaouache for the grain B2 don't correspond with our calculus.

b) For the grain $\mathrm{C} 1$ of the sample $\mathrm{P} 1$, the rolling plane crystalline direction reported by Kaouache generate an intersection of habit plane and the observation surface which is rotated an angle of 84.732 degrees.

On other hand, was made an analysis which consists in the calculation of next paths for both samples and all variants of each grain studied by Kaouache:

a) The vector field associated to the martensite variant selected

b) The distortion of a mark draws on the observation surface

For getting the last, was employed a mathematical model developed previously. This model, consist in a non homogenous displacement vector field which is capable to simulate the distortion of the material during a martensite variant formation [2]. Then the function is subject to a vector basis-change mathematical's operation for to project the function in to the observation system. The model is based in the basic principles established by Patoor for the martensitic transformation in shape memory materials [3].The model is shown next:

$$
\begin{gathered}
u\left(x_{T}, y_{T}, z_{T}\right)=\left(\begin{array}{c}
\delta f h w\left(y_{T}\right) \\
0 \\
0
\end{array}\right) \\
w\left(y_{T}\right)=\frac{f h+\sqrt{r_{0}^{2}+y_{T}^{2}}-\sqrt{r_{0}^{2}+\left(y_{T}-f h\right)^{2}}}{2 f h} \\
\left(x_{T}, y_{T}, z_{T}\right)^{T}=A_{X O \rightarrow X T}\left(x_{O}, y_{O}, z_{O}\right)^{T} \text { and } u\left(x_{O}, y_{O}, z_{O}\right)=A_{X T \rightarrow X O} u\left(x_{T}, y_{T}, z_{T}\right)
\end{gathered}
$$

where:

$\left(x_{T}, y_{T}, z_{T}\right)$ and $\left(x_{O}, y_{O}, z_{O}\right)$ are the coordinates of a point in the transformational system and in the observation system respectively

$A_{X O \rightarrow X T}$ and $A_{X T \rightarrow X O}$ are the vector basis change tensor from observation to transformation basis and from transformation to observation basis, which are determinates if we know the transformational system and the sample crystalline orientation.

The model allow us, determined the grain distortion mode which can be related with the interaction between neighbor grains. Also the model shows, the displacement direction which can be indicate the stress applied direction to a grain on other. All these information can't be obtained by the factor "Schmid Factor" criteria.

Finally, employing the criteria reported by Bucheit et al[3 and 4] were calculated the transformation diagrams for a plane-stress state for each grain analysed by Kaouache et al [1]. The diagrams were building by the calculation of the Taylor factor for each one of martensite variants under de simple tension stress on two perpendicular directions of the grain; one along to the rolling direction and the other perpendicular to it. Under this criteria also were found some interesting dates.

Although the in situ X-ray diffraction study made by Kaouache, indicate non presence of the normal stress different to the normal stress induced by the external load $\left(\sigma_{11}\right)$, the transformation diagrams shows proofs that the apparition of new martensite variants could be due to the presence of perpendicular normal stress $\left(\sigma_{33}\right)$ in tension or in compression. Particularity, the apparition of variant V4 corresponding of grain B1 (sample P1) reported by Kaouache et al [1] could be more easy induced by the application of $\sigma_{33}$ because request less energy. 


\section{Results}

\subsection{General parameters}

In the Table 1 is shows the Schimd Factor for the two variants induced in the grain B2 reported by Kaouache et al [ ] and these calculated here. As we can see, the values present some dispersion but any way they are the maximum values for the grain and therefore don't cause hard chances in the results.

Table 1. Schmid Factor values reported by Kaouache et al [1] and calculated in this work for the grain B2.

\begin{tabular}{|c|c|c|c|c|}
\hline Sample & Grain & Variant & FS reported & FS calculated \\
\hline \multirow{2}{*}{ P2 } & \multirow{2}{*}{ B2 } & V23 & 0.40 & 0.385 \\
\cline { 3 - 5 } & V18 & 0.38 & 0.395 \\
\hline
\end{tabular}

On other hand in the Figure 1(a) is show the intersection of the habit whit the observation surface for the grain $\mathrm{C} 1$, of the sample P1, employing the date of rolling plane (RP) and rolling direction (RD) reported by Kaouache et al [ ] it is $\mathrm{RP}=(0.84,0.13,0.52)$ and $\mathrm{RD}=[0.27,-0.94,-0.16]$. Them in the Figure $1 \mathrm{~b}$, is shows the same parameter calculated using as rolling plane equal to the negative value reported it is $\mathrm{RP}=(-0.84,-0.13,-0.52)$. It is clear the consistent of the result whit the experimental result employing the second case.

Is important to say that the Schmid Factor for all transformation elements is equal for both cases discusses before may be it was the reason of the mistake.

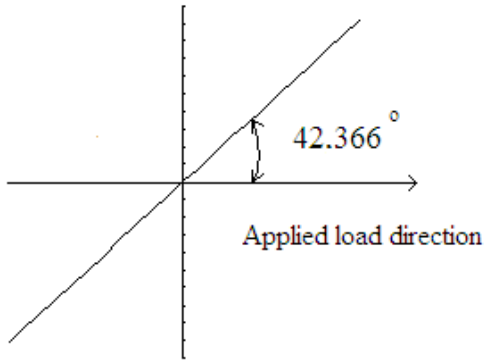

(a)

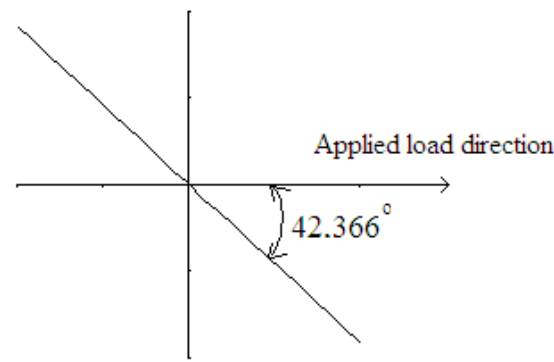

(b)

Fig. 1. a) Intersection between the corresponding habit plane and the observation surface take in count the rolling plane reported [1], b) Same parameter take in count the negative of the rolling plane reported.

\subsection{Simulation}

In the Figures 2 to 4 , it is show the transformational diagrams for plane-stress state, the intersection of the each habit plane with the observation surface, the distortion of a circular mark on the observation surface and the displacement field associated for the variants induced in each grain for the samples P1 and P2 reported by Kaouache.

As shows the Figures 2 and 3, corresponding with the grains of sample P1, the most variants present a vertical displacement component spatially in the grain $\mathrm{C} 1$ it seems to induce this displacement path to the others grains. As is clear in the transformation diagrams in the three cases is possible to induce a new variant by the apparition of normal stresses $\sigma_{33}$; V22 in the grain C1, V21 in B1 grain and V24 and V8 in the grain A1. A special case is present in the grain B1 where the variant V4 request a compression stress $\sigma_{33}$ whose value is similar to the stress $\sigma_{11}$ induced by the applied load. Is clear that non $\sigma_{33}$ stresses are applied on the samples and therefore they are induced by the interaction of near grains. Under this criteria the compression stress induced on the B1 grain could be due to the neighbors which don't had transformed on left to the grain.

Is important to say that non $\sigma_{33}$ stress, were identifying by the application of X-Ray diffraction technique. Nevertheless the transformational diagrams guaranties minimum energy criteria which is a physical principle it should be completed. Also as is well know the stress is a field and them it can change from point to point and the technique could not be capable to measure with enough precision. 


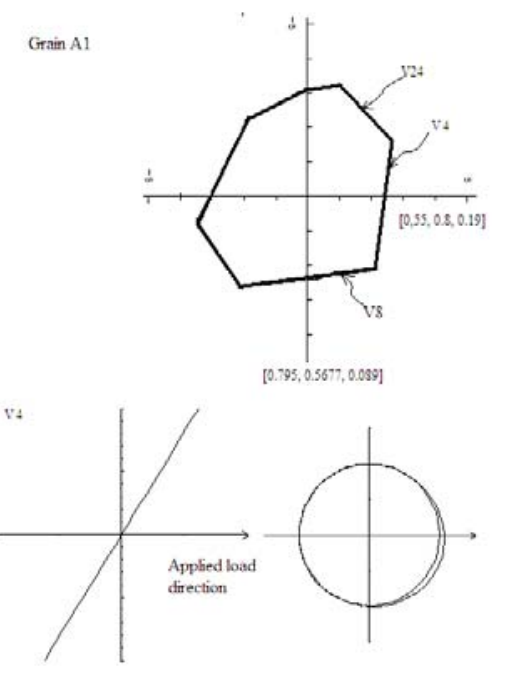

(a)

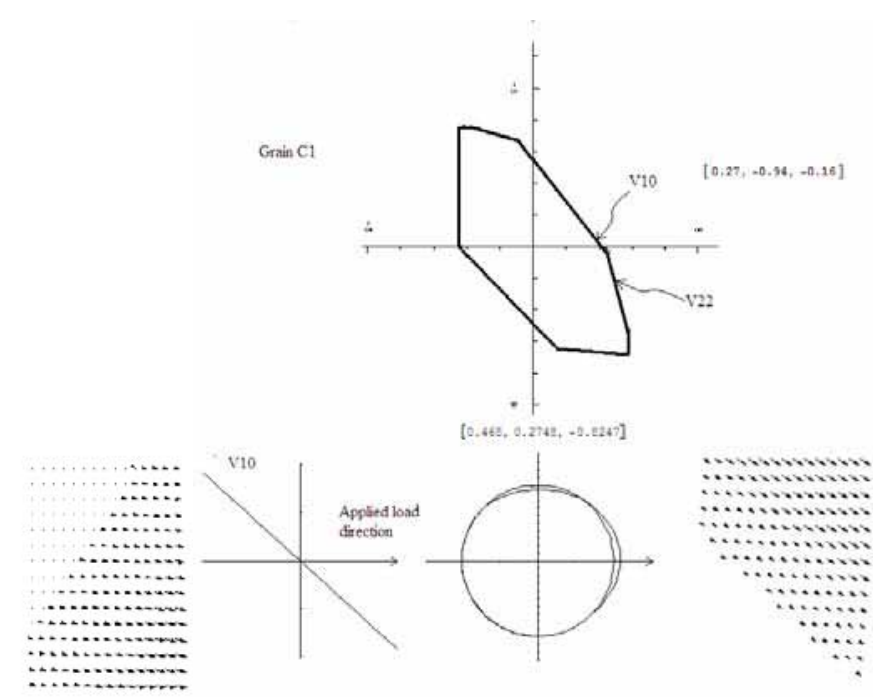

(b)

Fig. 2. Transformational diagram for plane-stress state, intersection of the habit plane whit the observation surface, distortion of a circular mark on the observation surface and the displacement field associated for the variants induced in a) grain $\mathrm{A} 1$ and b) grain $\mathrm{C} 1$.

Finally, the paths corresponding with the grain A2 shows similar detail to the grains in the sample P1. Certain tendency to lateral displacement is appreciated and the variants induced are located near in the transformation diagram both

\section{Conclusions}

The analysis show above shows the next:

a) The importance of the transformational diagram for plane-stress state and the simulation of the distortion during the transformation and the displacement field associated for the interpretation of the results analysed.

b) Under the criteria discussed in the present work the interaction between neighbor's grains it is manifested by the apparition of vertical stresses.

\section{References}

[1] B. Kaouache, K. Inal, S. Berveiller, A. Eberhardt and E. Patoor. Materials Science and Engineering A. 438-440, 773 (2006)

[2] J. Cortés-Pérez. Modelación matemática de transformación martensitica inducida por esfuerzo. Tésis doctoral. Programa de maestría y doctorado en Ingeniería, Facultad de Ingeniería, UNAM. México (2007)

[3] Patoor E., Berveiller M. Les alliages à mémoire de forme, Technologies de pointe. Hermes, PARIS, 1990.

[4] T. E. Buchheit and J. A. Wert.. Metallurgical and Materials Transactions A. 25, 2383 (1994).

[5] T. E. Buchheit, S. L. Kumpf and J. A. Wert. Acta metall. mater.43,4189 (1995). 

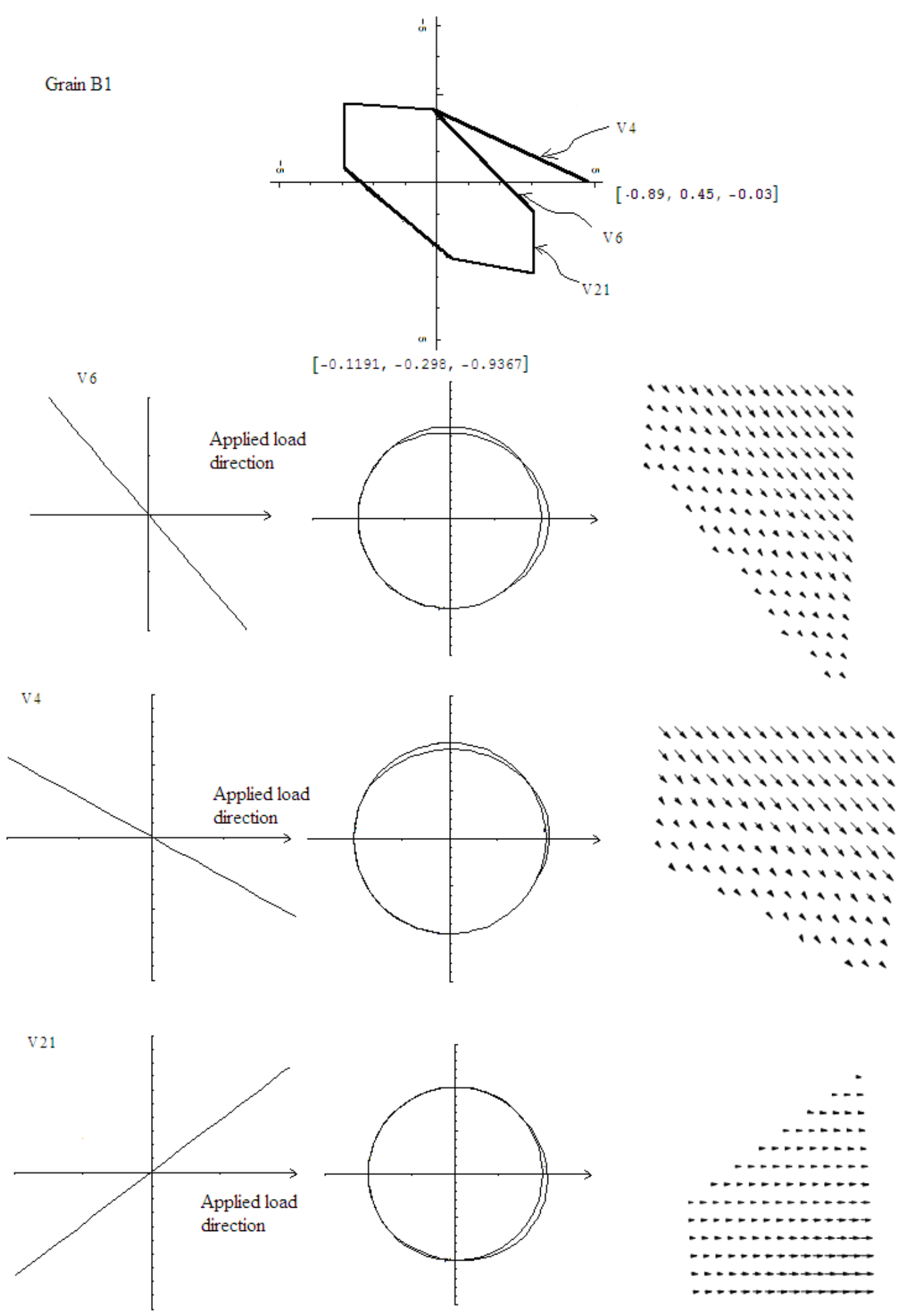

Fig. 3. Transformational diagram for plane-stress state, intersection of the habit plane whit the observation surface, distortion of a circular mark on the observation surface and the displacement field associated for the variants induced in grain B1. 


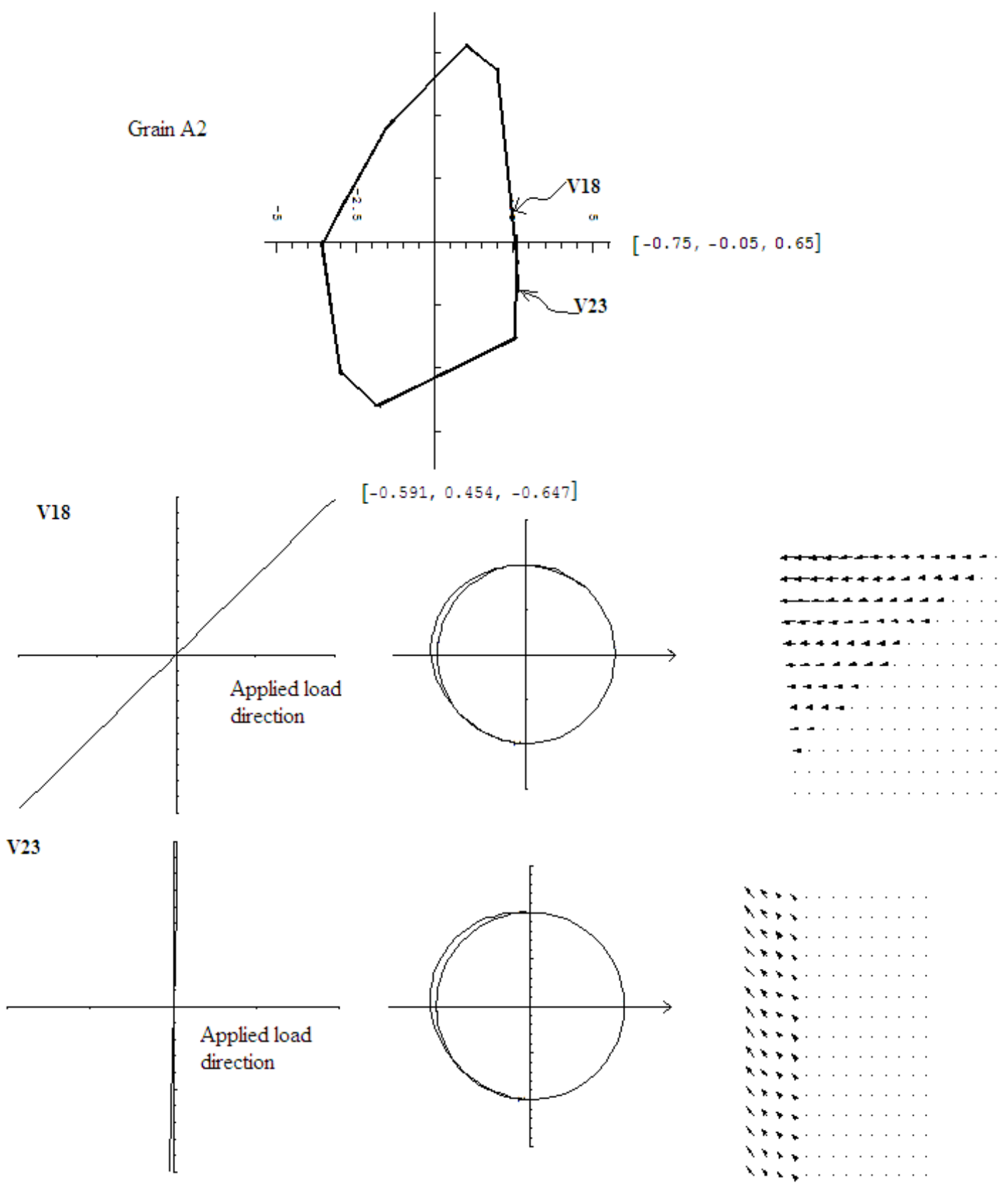

Fig. 4. Transformational diagram for plane-stress state, intersection of the habit plane whit the observation surface, distortion of a circular mark on the observation surface and the displacement field associated for the variants induced in grain $\mathrm{A} 2$. 